\title{
Connotation of Enterobacter cloacae-W6 and Serratia ficaria-W10 with or without carriers for improving growth, yield and nutrition of wheat
}

\author{
Muhammad Zafar-ul-Hye ${ }^{I^{*},}$,Umar Aslam ${ }^{\text {I, }}$ Bushra Muqaddas ${ }^{1}$ and Muhammad Baqir Hussain ${ }^{2}$ \\ ${ }^{1}$ Department of Soil Science, Bahauddin Zakariya University Multan, Pakistan. \\ ${ }^{2}$ Institute of Soil and Environmental Sciences, University of Agriculture Faisalabad, Pakistan.
}

\begin{abstract}
A pot experiment was conducted to compare the influence of Enterobacter cloacae-W6 and Serratia ficariaW10 (exopolysaccharides and auxin producing, phosphate solubilizing and abundantly root colonizing bacteria) inoculation either alone or in combination with or without carriers (peat, biogas slurry, press mud) on the growth, yield and nutrient concentration of wheat in semi-arid region of Multan. The experiment was arranged in completely randomized design with three replications. Results demonstrated significantly higher plant height, root/ spike length, shoot/ root dry weight, number of tillers/ spikelets/ grains, 1000 grain weight, grain/ straw/ biological yield, nitrogen/ phosphorus/ potassium concentrations of shoot and grain due to inoculation with W6 or W10 alone or in combination as compared to no inoculation. Carriers significantly lowered the spike length, root dry weight, grain number, biological/ grain/ straw yield, nitrogen/ phosphorus/ potassium concentration in shoot and nitrogen concentration in grains as compared to no carrier (seeds soaked for $1 \mathrm{~h}$ ). However, peat was statistically at par with no carrier for plant height, root length, shoot dry weight, number of tillers/spikelets, 1000 grain weight, grain phosphorus and potassium concentrations. Combined $($ W6 $\times$ W10) inoculation remained most prominent followed by W6 and W10, respectively. Similarly, inoculation without carrier (soaking) remained most efficient from carriers followed by peat as a carrier. Biogas slurry and press mud remained statistically at par with each other but lower than no carrier and peat in all parameters. Keeping in view the results, it can be inferred that combined inoculation without carrier (soaking) is most beneficial but peat can be recommended as best carrier from biogas slurry and press mud.
\end{abstract}

Keywords: Carriers, inoculation, seed soaking, wheat, production, nutrition

\section{Introduction}

Since the discovery of soil microorganisms in $18^{\text {th }}$ century, they have been used for crop production extensively (Mahmood et al., 2016). These plant beneficial bacteria have been termed as plant growth promoting rhizobacteria (PGPR) due to their plant growth improving activities in the rhizosphere (Kloepper and Schroth, 1978). Mainly plant beneficial microorganisms trigger plant growth through the production of phytohormones, increase the supply of nutrient via nitrogen fixation or phosphate solubilization, save plants from pathogens, improve soil physical conditions and rescue plant under abiotic stress in soil (Burd et al., 2000; Glick, 2003; Dey et al., 2004; Zaidi et al., 2006; Podile and Kishore, 2006; Saravanakumar et al., 2008).

Soil fertility and plant health depends on plant-microbe interaction under given soil conditions (Jeffries et al., 2003). Sustainable agriculture has coined the exploitation of plant beneficial rhizobacteria to increase the bioavailability of nutrient present in soil (Hayat et al., 2010). Number of bacteria have been explored for improving plant growth such as Rhizobium, Azotobacter, Pseudomonas, Azospirillum, Serratia, Bacillus, Enterobacter, Klebsiella, and Burkhuderia spp. (Staley and Drahos, 1994; Naveed et al., 2014a,b; Hussain et al., 2016; Hussain et al., 2017; Shahzad et al., 2017). Plant growth promoting bacteria are categorized as biofertilizers, biostimulators, and bioprotectors on the basis of their dominating plant beneficial attribute. Biofertilizers increase bioavailability and nutrient uptake in plant by increasing root growth and the production of siderophores and organic acids (Meyer, 2000; Ahmadi and Siosemardeh, 2005; Mehboob et al., 2012). Biostimulators modulate plant growth by auxin, cytokinin, gibberellin and abscisic acid production (Upadhyaya et al., 1991; Frankenberger and Arshad, 1995; Roy and Basu, 2004; Chi et al., 2005). Bioprotectors induce stress (biotic and abiotic) tolerance or resistance in plants with the production of antibiotics, siderophores, $\mathrm{HCN}$, exopolysaccharides and chitinases (Antoun et al., 1978; Kloepper et al., 1980; Liu et al., 1995; Antoun and Prevost, 2000; Neeraja et al., 2010; Hussain et al., 2014). The sole inoculation of PGPR strain may perform better if it could be connoted with other beneficial strain.

Beneficial effects of PGPR inoculation have been worked out with different strains for various crops. They postulated that the survival and colonization of inoculated bacteria in rhizosphere soil reduced due to various abiotic

*Email: zafarulhyegondal@yahoo.com 
and biotic factors (Campbell and Ephgrave, 1983; Heijnen et al., 1990). Therefore, method of PGPR inoculation could play a pivotal role in exploiting the plant growth promoting potential of the strain. Seed soaking in PGPR suspension and seed coating are the most efficient methods of inoculation however, the cost and availability of materials required are main cause of concern. In seed coating a suspension of PGPR with a solid and a sticking agent is applied on the seed to cover it uniformly (Bardin and Huang, 2003). Inoculated seeds exposed to environment cause cell death and reduce the population of beneficial microorganism. Use of carrier materials for delivery of optimal cell number, adhesive agent for sticking of bacteria on seed and desiccation eluding material for inoculum is recommended (Elegba and Rennie, 1984). Therefore, different carriers are used to prolong the viability of potential PGPR on the inoculated seed with higher population size (Chao and Alexander, 1984; Elegba and Rennie, 1984). It is obvious that the population of desired bacteria in rhizosphere soil solely depends on the load of inoculum applied (Milus and Rothrock, 1993). The more population of desired bacteria on seed can increase the probability of quenching benefits (Hebbar et al., 1992).

Keeping in view the whole discussion, present investigation was designed to explore the connoted effect of Enterobacter cloacae-W6 and Serratia ficaria-W10 on wheat crop along with different carrier materials (peat, biogas slurry, press mud) or soaking in bacterial suspension.

\section{Materials and methods}

\section{Inoculum and inoculation}

Two potential plant growth promoting rhizobacterial strains Enterobacter cloacae-W6 and Serratia ficaria-W10 (exopolysaccharides and auxin producing, phosphate solubilizing and abundantly root colonizing bacteria) (Nadeem et al., 2010) were acquired from Soil Microbiology and Biochemistry Laboratory, Institute of Soil and Environmental Sciences, University of Agriculture, Faisalabad, Pakistan. The strains were refreshed in Soil Microbiology Laboratory, Faculty of Agricultural Sciences and Technology, Bahauddin Zakariya University, Multan using sterilized $50 \mathrm{~mL}$ growth media in a $100 \mathrm{~mL}$ conical flask (Dworkin and Foster, 1958). The inoculated media was incubated for $72 \mathrm{~h}$ on an orbital shaking tray at $110 \mathrm{rpm}$ and at room temperature. The optical density (OD) of inocula was maintained at $0.5\left(\approx 10^{6}\right.$ cells $\left.\mathrm{mL}^{-1}\right)$ using densitometer.

Freshly prepared inocula of W6 and W10 was used for seed inoculation of wheat cultivar FSD-2008 either alone or in combination (W6×W10, 1:1 by volume) using sterilized peat, biogas slurry, or sugarcane press mud as carriers and $12 \%$ sugar solution as sticking agent. Inocula of each bacteria was mixed with carrier at 2:10 ratio (v/w). Seed and carrier based inocula mixed at 40:1 (w/w). Sugar solution was sprayed on the seed and mixed with carrier based inocula till uniform coating. Un-inoculated control was maintained by coating seeds with sterilized broth and carriers. Inoculated and un-inoculated control without carrier was developed by soaking wheat seeds in bacterial broth for $60 \mathrm{~min}$. The coated seeds dried for 6 to $8 \mathrm{~h}$ in shade.

\section{Pot trial setup}

A pot experiment was designed with inoculation (Factor A) and carrier material (Factor B) as factors. Soil for the experiment was collected from the surface layer of the field at University Agriculture Farm, Bahauddin Zakariya University, Multan. About $10 \mathrm{~kg}$ of dried, sieved and analyzed soil (sand $50 \%$, silt $27 \%$, clay $22 \%$, EC $3 \mathrm{dS} \mathrm{m}^{-1}$, $\mathrm{pH} 8.7$, total $\mathrm{N} 0.08 \%$, available $\mathrm{P} 7.23 \mathrm{mg} \mathrm{kg}^{-1}$, and extractable $\mathrm{K} 97 \mathrm{mg} \mathrm{kg}^{-1}$ ) was filled in each pot. The pots were arranged following completely randomized design with three replications. Treated seeds of wheat cultivar FSD-2008 were sown in pots ( 9 seeds per pot). After germination, the pots thinned to maintain three uniformly germinated plants per pot. Recommended dose of fertilizer urea, diammonium phosphate and muriate of potash (N:P:K at 120:90:60 kg ha' $\left.{ }^{1}\right)$ were applied in each pot. Phosphorus and potassium were applied as basal whereas nitrogen was applied in three splits (1/3 basal, 1/3 tillering, 1/3 flowering). Tube well water available at the farm was irrigated to fulfill the water requirements of the crop. Plants were grown up to maturity and at harvest parameters relating to growth, yield and nutrient concentrations were recorded.

\section{Nutrient analysis}

Dried and ground plant and grain samples were digested following wet digestion method described by Wolf (1982) using di-acid mixture $\left(\mathrm{H}_{2} \mathrm{O}_{2}\right.$ and conc. $\mathrm{H}_{2} \mathrm{SO}_{4}$ at $1: 2$ proportion). The digested sample solution filtrate was distillated using Kjeldahl apparatus and back titrated with dilute $\mathrm{H}_{2} \mathrm{SO}_{4}(0.01 \mathrm{~N})$ to calculate the nitrogen concentration (Jackson, 1962.). For phosphorus determination, the filtrate $(5 \mathrm{~mL})$ was mixed with Barton reagent $(10 \mathrm{~mL})$ and measured spectrophotometric readings at $420 \mathrm{~nm}$ wavelength (Ryan et al., 2001). Flame photometer was used to determine the concentration of potassium in digested samples (Ryan et al., 2001). The concentrations of phosphorus and potassium were calculated using standard curves.

\section{Statistical analysis}

The data collected was analyzed statistically following two analysis of variance in factorial settings. The treatment means were compared with least significant difference at 5\% probability (Steel et al., 1997). 


\section{Results}

\section{Growth parameters}

The interactions of carriers and inoculation demonstrated variable results in interaction. Therefore, main results have been described for all the parameters as following.

Inoculation with combination of PGPR strains (W6×W10) proved significantly efficient for improving plant height (Table 1) as compared to the separate W10 inoculation and at par with W6 inoculation, though all the inoculations remained significantly higher over un-inoculated control. Among the carriers, peat was significantly prominent as compared to biogas slurry and press mud but statistically at par with no carrier.
No carrier and peat as carrier remained statistically similar to each other but significantly different from biogas slurry and press mud for improving root length (Table 1). Un-inoculated control showed significantly lower root length as compared to PGPR inoculation whether alone W6 or W10 or in combination $\mathrm{W} 6 \times \mathrm{W} 10)$. The combined inoculation still showed highest increase in root length as compared to alone inoculation.

Dry weight of root (Table 1) varied significantly due to inoculation (either alone (W6 or W10) or in combination (W6 $\times$ W10) over un-inoculated control. Both the PGPR strains and their combination were statistically different in improving root dry weight where highest increase over control was given by combination (W6×W10) followed by W6 and W10,

Table 1: Influence of PGPR with carriers on the growth parameters of wheat

\begin{tabular}{|c|c|c|c|c|c|c|c|c|c|c|}
\hline & \multicolumn{2}{|c|}{ Plant height (cm) } & \multicolumn{8}{|c|}{ Root length $(\mathrm{cm})$} \\
\hline & $\begin{array}{l}\text { No } \\
\text { carrier }\end{array}$ & Peat & $\begin{array}{l}\text { Biogas } \\
\text { slurry }\end{array}$ & $\begin{array}{l}\text { Press } \\
\text { mud }\end{array}$ & Mean & $\begin{array}{l}\text { No } \\
\text { carrier }\end{array}$ & Peat & $\begin{array}{l}\text { Biogas } \\
\text { slurry }\end{array}$ & $\begin{array}{l}\text { Press } \\
\text { mud }\end{array}$ & Mean \\
\hline $\begin{array}{l}\text { No } \\
\text { inoculation }\end{array}$ & $49.2 \mathrm{~h}$ & $56 \mathrm{~g}$ & $61.3 \mathrm{f}$ & $58 \mathrm{fg}$ & $56.13 \mathrm{c}$ & $8.1 \mathrm{ef}$ & $7.96 \mathrm{f}$ & $8.26 \mathrm{def}$ & $6.76 \mathrm{~g}$ & $7.77 \mathrm{c}$ \\
\hline W6 & $72.3 \mathrm{ab}$ & 72 abc & $67 \mathrm{de}$ & $65.7 \mathrm{e}$ & $69.25 \mathrm{ab}$ & $9.2 \mathrm{bc}$ & $9.76 \mathrm{~b}$ & $8.23 \mathrm{ef}$ & 8.23 ef & $8.86 \mathrm{~b}$ \\
\hline W10 & $70.2 \mathrm{bcd}$ & $70.3 \mathrm{bcd}$ & $65.2 \mathrm{e}$ & 68.7 b-e & $68.61 \mathrm{~b}$ & $9.44 \mathrm{bc}$ & $9.26 \mathrm{bc}$ & $9.16 \mathrm{bcd}$ & $8.83 \mathrm{c}-\mathrm{f}$ & $9.17 \mathrm{~b}$ \\
\hline W6xW10 & $75 \mathrm{a}$ & $72.3 \mathrm{ab}$ & $67.5 \mathrm{de}$ & $68.3 \mathrm{cde}$ & $70.77 \mathrm{a}$ & $12.73 \mathrm{a}$ & $12.53 \mathrm{a}$ & $9.0 \mathrm{~b}-\mathrm{e}$ & $9.5 \mathrm{bc}$ & $10.94 \mathrm{a}$ \\
\hline \multirow[t]{2}{*}{ Mean } & $66.68 \mathrm{ab}$ & $67.65 \mathrm{a}$ & $65.27 \mathrm{~b}$ & $65.16 \mathrm{~b}$ & & $9.87 \mathrm{a}$ & $9.88 \mathrm{a}$ & $8.66 \mathrm{~b}$ & $8.33 \mathrm{~b}$ & \\
\hline & \multicolumn{5}{|c|}{ Shoot dry weight (g) } & \multicolumn{4}{|c|}{ Root dry weight (g) } & \\
\hline $\begin{array}{l}\text { No } \\
\text { inoculation }\end{array}$ & $0.59 \mathrm{ij}$ & $0.55 \mathrm{j}$ & $0.61 \mathrm{ij}$ & $0.76 \mathrm{hi}$ & $0.63 \mathrm{c}$ & $0.43 \mathrm{f}$ & $0.33 \mathrm{~g}$ & $0.56 \mathrm{e}$ & $0.39 \mathrm{fg}$ & $0.43 \mathrm{~d}$ \\
\hline W6 & $1.22 \mathrm{~b}-\mathrm{e}$ & $1.36 \mathrm{~b}$ & $1.27 \mathrm{~b}-\mathrm{e}$ & $1.28 \mathrm{bcd}$ & $1.28 \mathrm{a}$ & $0.67 \mathrm{~d}$ & $0.87 \mathrm{~b}$ & $0.65 \mathrm{de}$ & $0.46 \mathrm{f}$ & $0.66 \mathrm{~b}$ \\
\hline W10 & $1.16 \mathrm{c}-\mathrm{f}$ & $1.32 \mathrm{bc}$ & $0.87 \mathrm{gh}$ & $1.16 \mathrm{c}-\mathrm{f}$ & $1.13 \mathrm{~b}$ & $0.76 \mathrm{c}$ & $0.38 \mathrm{fg}$ & $0.57 \mathrm{e}$ & $0.64 \mathrm{de}$ & $0.59 \mathrm{c}$ \\
\hline W6 $\times W 10$ & $1.65 \mathrm{a}$ & $1.12 \mathrm{def}$ & $1.09 \mathrm{ef}$ & $0.99 \mathrm{fg}$ & $1.21 \mathrm{ab}$ & $0.98 \mathrm{a}$ & $0.93 \mathrm{ab}$ & $0.69 \mathrm{~cd}$ & $0.69 \mathrm{~cd}$ & $0.82 \mathrm{a}$ \\
\hline \multirow[t]{2}{*}{ Mean } & $1.16 \mathrm{a}$ & $1.09 \mathrm{ab}$ & $0.96 \mathrm{c}$ & $1.05 \mathrm{c}$ & & $0.71 \mathrm{a}$ & $0.63 \mathrm{~b}$ & $0.62 \mathrm{~b}$ & $0.55 \mathrm{c}$ & \\
\hline & \multicolumn{3}{|c|}{ Spike length $(\mathrm{cm})$} & \multicolumn{7}{|c|}{ Number of tillers per plant } \\
\hline $\begin{array}{l}\text { No } \\
\text { inoculation }\end{array}$ & $5.86 \mathrm{~h}$ & $5.73 \mathrm{~h}$ & $5.73 \mathrm{~h}$ & $5.80 \mathrm{~h}$ & $5.78 \mathrm{c}$ & $2.3 \mathrm{~d}$ & $2.3 \mathrm{~d}$ & $2.3 \mathrm{~d}$ & $2.3 \mathrm{~d}$ & $2.3 \mathrm{~b}$ \\
\hline W6 & $9.66 \mathrm{ab}$ & $8.80 \mathrm{~cd}$ & $7.63 \mathrm{fg}$ & $8.40 \mathrm{de}$ & $8.61 \mathrm{a}$ & $4.3 \mathrm{a}$ & $4.0 \mathrm{ab}$ & $3.0 \mathrm{~cd}$ & $3.0 \mathrm{~cd}$ & $3.6 \mathrm{a}$ \\
\hline W10 & $9.23 \mathrm{bc}$ & 8.00 ef & $7.66 \mathrm{f}$ & $7.66 \mathrm{f}$ & $8.14 \mathrm{~b}$ & $4.3 \mathrm{a}$ & $4.0 \mathrm{ab}$ & $3.0 \mathrm{~cd}$ & $3.0 \mathrm{~cd}$ & $3.6 \mathrm{a}$ \\
\hline W6xW10 & $9.83 \mathrm{a}$ & $9.13 \mathrm{c}$ & $8.26 \mathrm{e}$ & $7.20 \mathrm{~g}$ & $8.62 \mathrm{a}$ & $4.7 \mathrm{a}$ & $4.3 \mathrm{a}$ & $3.3 \mathrm{bc}$ & $3.3 \mathrm{bc}$ & $3.9 \mathrm{a}$ \\
\hline Mean & $8.65 \mathrm{a}$ & $7.92 \mathrm{~b}$ & $7.32 \mathrm{c}$ & $7.27 \mathrm{c}$ & & $3.9 \mathrm{a}$ & $3.7 \mathrm{a}$ & $2.9 \mathrm{~b}$ & $2.9 \mathrm{~b}$ & \\
\hline
\end{tabular}

Means sharing similar letters are statistically at par with each other at $5 \%$ probability level.

Shoot dry weight (Table 1) was significantly increased by the inoculation of W6 or W10 alone or in combination (W6×W10) as compared to un-inoculated control. Maximum shoot dry weight was observed in W6 inoculated plants followed by combination $(\mathrm{W} 6 \times \mathrm{W} 10)$ which remained statistically similar to W10 inoculation. Inoculation without carriers (liquid dip) and peat as a carrier showed significant increase in shoot dry weight as compared to biogas slurry or press mud based inoculation. However, the peat based inoculation was non-significant as compared to no carrier treatment. respectively. Carriers (peat, biogas slurry and press mud) decreased root dry weight significantly in comparison to no carrier.

Spike length (Table 1) increased significantly with the inoculation of $\mathrm{W} 6, \mathrm{~W} 10$ alone and their combination (W6 $\times$ W10) in contrast to no inoculation but the performance with liquid or no carrier based inoculation was significantly higher over carrier (peat, biogas slurry, press mud) based inoculation. 
The number of tillers per plant (Table 1) were equally increased by the inoculation with W6 or W10 alone or in combination (W6 $\times \mathrm{W} 10)$ as compared to un-inoculated control. Only, peat based inoculation remained statistically similar to no carrier inoculation but these both were statistically prominent from biogas slurry and press mud based inoculation.

\section{Yield parameters}

The inoculation with W6 and W10 significantly improved number of spikelets per spike (Table 2) as compared to un-inoculated control both in alone and in combination. Where W6 alone and combination (W6 $\times$ W10) showed significantly higher spikelets per spike over W10 inoculation and remained statistically similar to each other. Peat from carriers was statistically at par with no carrier though both were produced significantly higher spikelets per spike from biogas slurry and press mud based inoculation. carriers were significantly lower than no carrier but peat was prominent within carrier materials.

Thousand grain weight (Table 2) was increased significantly by the inoculation of W6 or W10 or combination as compared to un-inoculated control though differences among inoculants remained non-significant. Whereas carriers could not show significant improvement in thousand grain weight as compared to each other but were significantly lower than no carrier.

The combined $(\mathrm{W} 6 \times \mathrm{W} 10)$ inoculation with no carrier showed highest biological yield (Table 2) as compared to no inoculation and carriers. Inoculation with either W6 or W10 or combination was significantly higher as compared to un-inoculated control. Among carriers, the differences were significant but peat remained at top followed by biogas slurry and press mud, respectively.

Grain yield (Table 2) was significantly increased by

Table 2: Influence of PGPR with carriers on the yield parameters of wheat

\begin{tabular}{|c|c|c|c|c|c|c|c|c|c|c|}
\hline & \multicolumn{5}{|c|}{ Number of spikelets per spike } & \multicolumn{5}{|c|}{ Number of grains per spike } \\
\hline & $\begin{array}{c}\text { No } \\
\text { carrier }\end{array}$ & Peat & $\begin{array}{l}\text { Biogas } \\
\text { slurry }\end{array}$ & $\begin{array}{l}\text { Press } \\
\text { mud }\end{array}$ & Mean & $\begin{array}{c}\text { No } \\
\text { carrier }\end{array}$ & Peat & $\begin{array}{l}\text { Biogas } \\
\text { slurry }\end{array}$ & $\begin{array}{l}\text { Press } \\
\text { mud }\end{array}$ & Mean \\
\hline $\begin{array}{l}\text { No } \\
\text { inoculation }\end{array}$ & $8.4 \mathrm{f}$ & $9.06 \mathrm{ef}$ & $9.26 \mathrm{ef}$ & $9.2 \mathrm{ef}$ & $8.98 \mathrm{c}$ & $13.33 \mathrm{f}$ & $13.33 \mathrm{f}$ & $15 \mathrm{ef}$ & $14.33 \mathrm{f}$ & $14.00 \mathrm{c}$ \\
\hline W6 & $13.96 \mathrm{~b}$ & $15.1 \mathrm{a}$ & $12.7 \mathrm{c}$ & $12.06 \mathrm{~cd}$ & $13.46 \mathrm{a}$ & $22 \mathrm{~d}$ & $23.66 \mathrm{~cd}$ & $22.33 \mathrm{~d}$ & $18 \mathrm{e}$ & $21.50 \mathrm{~b}$ \\
\hline W10 & $14.06 \mathrm{ab}$ & $12.13 \mathrm{~cd}$ & $9.93 \mathrm{e}$ & $11.33 \mathrm{~d}$ & $11.86 \mathrm{~b}$ & $27.66 \mathrm{~b}$ & $22.33 \mathrm{~d}$ & $18 \mathrm{e}$ & $22.33 \mathrm{~d}$ & $22.58 \mathrm{~b}$ \\
\hline W6×W10 & $15.17 \mathrm{a}$ & $14.31 \mathrm{ab}$ & $12.55 \mathrm{c}$ & $12.33 \mathrm{~cd}$ & $13.59 \mathrm{a}$ & $39.66 \mathrm{a}$ & $28.66 \mathrm{~b}$ & $26.33 \mathrm{bc}$ & $23.33 \mathrm{~cd}$ & $29.50 \mathrm{a}$ \\
\hline \multirow[t]{2}{*}{ Mean } & $12.90 \mathrm{a}$ & $12.65 \mathrm{a}$ & $11.11 \mathrm{~b}$ & $11.23 \mathrm{~b}$ & & $25.66 \mathrm{a}$ & $22.00 \mathrm{~b}$ & $20.42 \mathrm{bc}$ & $19.50 \mathrm{c}$ & \\
\hline & \multicolumn{5}{|c|}{1000 grain weight $(\mathrm{g})$} & \multicolumn{5}{|c|}{ Biological yield $\left(\mathrm{g} \mathrm{pot}^{-1}\right)$} \\
\hline $\begin{array}{l}\text { No } \\
\text { inoculation }\end{array}$ & $28 \mathrm{f}$ & $28.3 \mathrm{f}$ & 30.7 ef & 31 ef & $29.50 \mathrm{~b}$ & 39.671 & $47 \mathrm{k}$ & $51 \mathrm{j}$ & $57.25 \mathrm{i}$ & $48.73 \mathrm{c}$ \\
\hline W6 & $42 \mathrm{ab}$ & $40 \mathrm{a}-\mathrm{d}$ & $31.3 \mathrm{ef}$ & $40 \mathrm{a}$ & $38.33 \mathrm{a}$ & $76.17 \mathrm{c}$ & $74.33 \mathrm{~d}$ & $67.67 \mathrm{f}$ & $60.25 \mathrm{~h}$ & $69.61 \mathrm{~b}$ \\
\hline W10 & 39 a-e & 31 ef & $32 \mathrm{c}-\mathrm{f}$ & $40.3 \mathrm{abc}$ & $35.58 \mathrm{a}$ & $75.87 \mathrm{~cd}$ & $65.88 \mathrm{~g}$ & $67.83 \mathrm{f}$ & $65.58 \mathrm{~g}$ & $68.79 \mathrm{~b}$ \\
\hline W6 $\times W 10$ & $47.7 \mathrm{a}$ & $36.7 \mathrm{~b}-\mathrm{f}$ & $32.7 \mathrm{c}-\mathrm{f}$ & $32 \mathrm{c}-\mathrm{f}$ & $37.25 \mathrm{a}$ & $116.12 \mathrm{a}$ & $86.9 \mathrm{~b}$ & $71.48 \mathrm{e}$ & $69.25 \mathrm{f}$ & $85.94 \mathrm{a}$ \\
\hline \multirow[t]{2}{*}{ Mean } & $39.17 \mathrm{a}$ & $34.00 \mathrm{~b}$ & $31.67 \mathrm{~b}$ & $35.83 \mathrm{ab}$ & & $76.96 \mathrm{a}$ & $68.53 \mathrm{~b}$ & $64.50 \mathrm{c}$ & $63.08 \mathrm{~d}$ & \\
\hline & \multicolumn{5}{|c|}{ Grain yield $\left(\mathrm{g} \mathrm{pot}^{-1}\right)$} & \multicolumn{5}{|c|}{ Straw yield $\left(\mathrm{g} \mathrm{pot}^{-1}\right)$} \\
\hline $\begin{array}{l}\text { No } \\
\text { inoculation }\end{array}$ & $10 \mathrm{k}$ & $12 \mathrm{j}$ & $15 \mathrm{i}$ & $15 \mathrm{i}$ & $13.00 \mathrm{~d}$ & $29.67 \mathrm{i}$ & $35 \mathrm{~h}$ & $36 \mathrm{~h}$ & $40.67 \mathrm{~g}$ & $35.34 \mathrm{c}$ \\
\hline W6 & $22.67 \mathrm{c}$ & $20.67 \mathrm{de}$ & 19.3efg & $18.7 \mathrm{fgh}$ & $20.34 \mathrm{~b}$ & $54.17 \mathrm{c}$ & $53 \mathrm{c}$ & $48.33 \mathrm{def}$ & $42.33 \mathrm{~g}$ & $49.46 \mathrm{~b}$ \\
\hline W10 & 20.05 ef & $18.5 \mathrm{gh}$ & $17.33 \mathrm{~h}$ & $17.33 \mathrm{~h}$ & $18.30 \mathrm{c}$ & $55.17 \mathrm{c}$ & $47.33 \mathrm{f}$ & $50.5 \mathrm{~d}$ & $48.25 \mathrm{ef}$ & $50.31 \mathrm{~b}$ \\
\hline $\mathrm{W6} 6 \mathrm{~W} 10$ & $28.17 \mathrm{a}$ & $25.92 \mathrm{~b}$ & $21.8 \mathrm{~cd}$ & $20.33 \mathrm{e}$ & $24.04 \mathrm{a}$ & $87.93 \mathrm{a}$ & $60.98 \mathrm{~b}$ & $49.73 \mathrm{de}$ & 49def & $61.91 \mathrm{a}$ \\
\hline Mean & $20.22 \mathrm{a}$ & $19.27 \mathrm{~b}$ & $18.35 \mathrm{c}$ & $17.83 \mathrm{c}$ & & $56.74 \mathrm{a}$ & $49.08 \mathrm{~b}$ & $46.14 \mathrm{c}$ & $45.06 \mathrm{c}$ & \\
\hline
\end{tabular}

Means sharing similar letters are statistically at par with each other at 5\% probability level.

The combination (W6 $6 \mathrm{~W} 10)$ of bacterial inoculation produced maximum number of grains per spike (Table 2) as compared to un-inoculated control and inoculated treatments (W6 or W10). Inoculation as a whole with W6 or W10 or combination significantly improved number of grains as compared to un-inoculated control. All the inoculation over no inoculation where combination (W6 $\times$ W10) showed highest grain yield followed by W6 and W10, respectively. No carrier again remained significantly higher as compared to carriers like peat, biogas slurry and press mud but peat among carriers was significantly at top. 
Inoculation with $\mathrm{W} 6, \mathrm{~W} 10$ and their combination produced significantly higher straw yield (Table 2) in comparison to no inoculation. Combined inoculation (W6 $\times \mathrm{W} 10$ ) was most prominent as compared to W6 or W10 inoculation alone. Peat as a carrier was most efficient in improving straw yield as compared to biogas slurry and press mud though no carrier treatment showed significantly higher straw yield over carriers.

\section{Nutrient concentrations}

Nitrogen concentration in shoot and grain (Table 3) increased significantly due to inoculation with W6, W10 or combination (W6 $\times \mathrm{W} 10)$ in contrast to no inoculation. The differences among the inoculation treatments also remained significant where (W6 $\times$ W10) was at the top followed by W6 and W10, respectively. However, no carriers produced significantly higher shoot or grain nitrogen concentration as compared to carriers (peat, biogas slurry and press mud). Peat remained prominent carriers followed by biogas slurry and press mud, respectively, for improving grain or shoot nitrogen concentration. observed in plants inoculated with combination (W6 6 W10). Grain and shoot phosphorus concentration in peat and no carrier was significantly higher as compared to biogas slurry or press mud. However, peat was statistically at par with no carrier for grain phosphorus and significantly lower for shoot phosphorus. Biogas slurry and press mud were nonsignificant to each other in both shoot and grain phosphorus concentrations. Combined (W6 $\times$ W10) inoculation produced maximum and significantly higher concentration of potassium (Table 3) in shoot and grain samples as compared to no inoculation and W6 or W10 inoculation. Even though no inoculation remained significantly lower in grain and shoot potassium concentration over W6 or W10 inoculation. Carriers showed significantly lower concentration of shoot potassium over no carrier but peat was statistically higher as compared to biogas slurry and press mud. Both press mud and biogas slurry were statistically similar for grain or shoot potassium concentrations. Peat and no inoculation were at par for improving grain potassium concertation but significantly higher from biogas slurry and press mud.

Table 3: Influence of PGPR with carriers on the nitrogen, phosphorus and potassium concentrations of wheat

\begin{tabular}{|c|c|c|c|c|c|c|c|c|c|c|}
\hline & \multicolumn{5}{|c|}{ Nitrogen in shoot (\%) } & \multicolumn{5}{|c|}{ Nitrogen in grains (\%) } \\
\hline & $\begin{array}{c}\text { No } \\
\text { carrier }\end{array}$ & Peat & $\begin{array}{l}\text { Biogas } \\
\text { slurry }\end{array}$ & $\begin{array}{l}\begin{array}{l}\text { Press } \\
\text { mud }\end{array} \\
\end{array}$ & Mean & $\begin{array}{c}\text { No } \\
\text { carrier }\end{array}$ & Peat & $\begin{array}{l}\text { Biogas } \\
\text { slurry }\end{array}$ & $\begin{array}{l}\text { Press } \\
\text { mud }\end{array}$ & Mean \\
\hline $\begin{array}{l}\text { No } \\
\text { inoculation }\end{array}$ & $1.05 \mathrm{k}$ & $1.15 \mathrm{j}$ & $1.2 \mathrm{i}$ & $1.18 \mathrm{ij}$ & $1.15 \mathrm{~d}$ & 1.571 & $1.75 \mathrm{k}$ & $1.8 \mathrm{j}$ & $1.75 \mathrm{k}$ & $1.72 \mathrm{~d}$ \\
\hline W6 & $1.63 \mathrm{c}$ & $1.6 \mathrm{c}$ & $1.49 \mathrm{ef}$ & $1.46 \mathrm{fg}$ & $1.55 \mathrm{~b}$ & $2.44 \mathrm{c}$ & $2.4 \mathrm{~d}$ & $2.23 \mathrm{f}$ & $2.17 \mathrm{gh}$ & $2.31 \mathrm{~b}$ \\
\hline W10 & $1.49 \mathrm{e}$ & $1.45 \mathrm{~g}$ & $1.43 \mathrm{~g}$ & $1.3 \mathrm{~h}$ & $1.42 \mathrm{c}$ & $2.23 \mathrm{f}$ & $2.17 \mathrm{gh}$ & $2.14 \mathrm{~h}$ & $1.95 \mathrm{i}$ & $2.12 \mathrm{c}$ \\
\hline $\mathrm{W6} \times \mathrm{W} 10$ & $1.85 \mathrm{a}$ & $1.71 \mathrm{~b}$ & $1.55 \mathrm{~d}$ & $1.46 \mathrm{efg}$ & $1.64 \mathrm{a}$ & $2.77 \mathrm{a}$ & $2.58 \mathrm{~b}$ & $2.32 \mathrm{e}$ & $2.2 \mathrm{fg}$ & $2.47 \mathrm{a}$ \\
\hline \multirow[t]{2}{*}{ Mean } & $1.51 \mathrm{a}$ & $1.48 \mathrm{~b}$ & $1.42 \mathrm{c}$ & $1.35 \mathrm{~d}$ & & $2.25 \mathrm{a}$ & $2.23 \mathrm{~b}$ & $2.12 \mathrm{c}$ & $2.02 \mathrm{~d}$ & \\
\hline & \multicolumn{5}{|c|}{ Phosphorus in shoot (\%) } & \multicolumn{5}{|c|}{ Phosphorus in grains (\%) } \\
\hline $\begin{array}{l}\text { No } \\
\text { inoculation }\end{array}$ & $0.2 \mathrm{k}$ & $0.23 \mathrm{j}$ & $0.21 \mathrm{k}$ & $0.24 \mathrm{i}$ & $0.22 \mathrm{~d}$ & $0.34 \mathrm{i}$ & $0.38 \mathrm{~h}$ & $0.37 \mathrm{~h}$ & $0.43 \mathrm{~g}$ & $0.38 \mathrm{~d}$ \\
\hline W6 & $0.35 \mathrm{c}$ & $0.34 \mathrm{~d}$ & $0.3 \mathrm{f}$ & $0.29 \mathrm{gh}$ & $0.32 \mathrm{~b}$ & $0.62 \mathrm{c}$ & $0.60 \mathrm{c}$ & $0.53 \mathrm{e}$ & $0.51 \mathrm{ef}$ & $0.57 \mathrm{~b}$ \\
\hline W10 & $0.34 \mathrm{~d}$ & $0.32 \mathrm{e}$ & $0.29 \mathrm{~g}$ & $0.28 \mathrm{~h}$ & $0.31 \mathrm{c}$ & $0.6 \mathrm{c}$ & $0.57 \mathrm{~d}$ & $0.51 \mathrm{ef}$ & $0.5 \mathrm{f}$ & $0.55 \mathrm{c}$ \\
\hline W6 6 W10 & $0.39 \mathrm{a}$ & $0.37 \mathrm{~b}$ & $0.34 \mathrm{~d}$ & $0.32 \mathrm{e}$ & $0.36 \mathrm{a}$ & $0.69 \mathrm{a}$ & $0.65 \mathrm{~b}$ & $0.6 \mathrm{c}$ & $0.57 \mathrm{~d}$ & $0.63 \mathrm{a}$ \\
\hline \multirow[t]{2}{*}{ Mean } & $0.32 \mathrm{a}$ & $0.31 \mathrm{~b}$ & $0.29 \mathrm{c}$ & $0.28 \mathrm{c}$ & & $0.56 \mathrm{a}$ & $0.55 \mathrm{a}$ & $0.50 \mathrm{~b}$ & $0.50 \mathrm{~b}$ & \\
\hline & \multicolumn{5}{|c|}{ Potassium in shoot (\%) } & \multicolumn{5}{|c|}{ Potassium in grains (\%) } \\
\hline $\begin{array}{l}\text { No } \\
\text { inoculation }\end{array}$ & $0.69 \mathrm{k}$ & $0.72 \mathrm{i}$ & $0.70 \mathrm{j}$ & $0.70 \mathrm{j}$ & $0.70 \mathrm{~d}$ & $1.33 \mathrm{i}$ & $1.36 \mathrm{i}$ & $1.34 \mathrm{i}$ & $1.36 \mathrm{i}$ & $1.35 \mathrm{~d}$ \\
\hline W6 & $0.93 \mathrm{~b}$ & $0.90 \mathrm{~d}$ & $0.87 \mathrm{e}$ & $0.85 \mathrm{f}$ & $0.89 \mathrm{~b}$ & $1.73 \mathrm{de}$ & $1.69 \mathrm{ef}$ & $1.65 \mathrm{fg}$ & $1.63 \mathrm{~g}$ & $1.68 \mathrm{~b}$ \\
\hline W10 & $0.88 \mathrm{e}$ & $0.85 \mathrm{f}$ & $0.80 \mathrm{~h}$ & $0.82 \mathrm{~g}$ & $0.84 \mathrm{c}$ & $1.61 \mathrm{~g}$ & $1.65 \mathrm{fg}$ & $1.57 \mathrm{~h}$ & $1.57 \mathrm{~h}$ & $1.60 \mathrm{c}$ \\
\hline W6×W10 & $0.97 \mathrm{a}$ & $0.93 \mathrm{~b}$ & $0.91 \mathrm{c}$ & $0.90 \mathrm{~d}$ & $0.93 \mathrm{a}$ & $1.88 \mathrm{a}$ & $1.83 \mathrm{~b}$ & $1.77 \mathrm{c}$ & $1.75 \mathrm{~cd}$ & $1.81 \mathrm{a}$ \\
\hline Mean & $0.87 \mathrm{a}$ & $0.85 \mathrm{~b}$ & $0.82 \mathrm{c}$ & $0.82 \mathrm{c}$ & & $1.64 \mathrm{a}$ & $1.63 \mathrm{a}$ & $1.58 \mathrm{~b}$ & $1.58 \mathrm{~b}$ & \\
\hline
\end{tabular}

Means sharing similar letters are statistically at par with each other at $5 \%$ probability level.

The concentration of phosphorus (Table 3) significantly increased over no inoculation by the inoculation of W6, W10 or combination $(\mathrm{W} 6 \times \mathrm{W} 10)$ in both grain and shoot samples. Highest phosphorus concentration in shoot and grain was

\section{Discussion}

Many studies have demonstrated the beneficial plantmicrobe interaction where they can be used effectively to 
increase nutrient use efficiency and rescue crop growth and productivity (Herrera et al., 1993; Requena et al., 1997). After a cautious review it was observed that about 25 to $65 \%$ increase in crop growth and yield parameters can be gained for different crops and under diverse environments (Mahmood et al., 2016). In the present study, inoculation with Enterobacter cloacae-W6 showed significant increase in growth and yield parameters and nutrient concentrations against un-inoculated plant where combined inoculation of Enterobacter cloacae-W6 and Serratia ficaria-W10 remained most prominent. These increases might be due to their capability to increase root growth with higher auxin production in the rhizosphere, exopolysaccharides production to increase nutrient and moisture availability to plant roots and sanctuary to beneficial microbes, phosphate solubilization to increase $\mathrm{P}$ uptake, and capability to massive colonization of plant roots (Principe et al., 2007; Nadeem et al., 2010), and most importantly the synergism of the two strains. Plant growth improving effects of beneficial bacteria are well versed and documented but the synergy of the PGPR strains is more effective in increasing different crop yields under varied soil conditions (Nadeem et al., 2013; Islam et al., 2014; Paulucci et al., 2015). The combined inoculation of PGPR may ameliorate the nutrient deficient conditions in soil and increase nutrient use efficiency while solubilizing the soil bound nutrients and ultimately increase crop productivity (Herrera et al., 1993; Requena et al., 1997).

The benefits of inoculated PGPR to seeds directly depend on the cell count (Mahmood et al., 2016). More populace is the spermosphere with beneficial bacteria higher will be the gains. The inoculation method and carriers influence the PGPR colonization on seeds. The seeds soaked with inoculum showed significantly higher increases in the growth, yield and nutrient parameters of wheat as compared to biogas slurry and press mud coated seeds but remained at par with peat based coating in most parameters. Press mud and biogas slurry coated seeds showed similar results in majority parameters. Soaking of the seed with PGPR broth accelerate physiological changes for ultimate germination (Anitha et al., 2013) and increase beneficial microbe number in spermosphere (Taylor and Harman, 1990). Higher numbers in the spermosphere benefit the germinated plants to gain vigor and better defense against diseases, improve plant growth leading to higher crop yield (Callan et al., 1990). Peat is the best carrier as compared to press mud and biogas slurry as it provides nutrition, surface area and moisture to inoculated microbes. Peat has always been a preference as a carrier for inoculant but its availability and composition is a major limitation (Boonkerd and Singleton, 2002). Therefore, we have to move towards easily available materials which may contribute in the optimal supply of PGPR to plant seeds. No doubt biogas slurry and press mud based inoculation is not much efficient as compared to seed soaking in inoculum or peat based inoculation even though they produced good results as compared to un-inoculated control. Even though carriers increase the viable population of inoculants on the seed, the results are inconsistent. Inoculated microbes have to be competitive enough to let down the indigenous microflora for root colonization (Hebbar et al., 1992).

Soaking of seed with combination of both strains (W6 $\times$ W10) produced highest significant results in growth, yield and nutrient parameters followed by peat based inoculation of combination. Peat based PGPR inoculation increased plant height, yield, nitrogen, phosphorus and potassium concentrations in leaves, root, shoot and grain samples (Dey et al., 2004; Nadeem et al., 2010, 2013). Auxin production ability of the inoculated strains might have increased the root growth and the area under its influence to explore for more nutrients (Principe et al., 2007). The results demonstrated the synergism of Enterobacter cloacae-W6 and Serratia ficaria-W10 strains without carrier (soaking) and carrier material which is helping them to flourish and exert positive impacts on wheat growth. Arora and coworkers (2014) have described the coexistence of Rhizobium and Pseudomonas in sawdust, bagasse and coriander husk with high numbers. These high cell numbers could be beneficial for improving the productivity of agricultural crops. In a similar study, Sarma et al. (2011) examined talcum and vermiculite based formulations of pseudomonas strains R62, R81 and Pi either in combinations or alone. They recorded a significant increases in growth and yield of tomato due to the application of $(\mathrm{R} 81 \times \mathrm{Pi})$ talcum based formulation.

Finally it can be concluded that the connotation of Enterobacter cloacae-W6 and Serratia ficaria-W10 is most beneficial for improving growth, yield and nutrient concentration in wheat if seed soaked (no carrier). However, peat is the best carriers as compared to biogas slurry and sugarcane press mud.

\section{References}

Ahmadi, A., and A. Siosemardeh. 2005. Investigation on the physiological basis of grain yield and drought resistance in wheat: Leaf photosynthetic rate, stomatal conductance and non-stomatal limitation. International Journal of Agriculture and Biology 7: 807-811.

Anitha, D., T. Vijaya, N.V. Reddy, N. Venkateswarlu, D. Pragathi, K.C. Mouli. 2013. Microbial endophytes and their potential for improved bioremediation and biotransformation: a review. Indo American Journal of Pharmaceutical Research 3: 6408-6417.

Antoun, H. and D. Prevost. 2000. PGPR activity of rhizobium with nonleguminous plants. In: Proceedings of the 5th International PGPR workshop, Oct. 29-Nov. 
03, Villa Carlos Paz, Argentina 2000, published online. DOI: $10.13140 / 2.1 .3093 .7609$

Antoun, H., L.M. Bordeleau, C. Gagnon. 1978. Antagonisme entre Rhizobium meliloti et Fusarium oxysporum en relation avec I'efricacit6 symbiotique. Canadian Journal of Plant Sciences 58: 75-78.

Arora, N.K., S. Tiwari and R. Singh. 2014. Comparative study of different carriers inoculated with nodule forming and free living plant growth promoting bacteria suitable for sustainable agriculture. Journal of Plant Pathology and Microbiology 5: 229.

Boonkerd, N. and P. Singleton. 2002. Production of rhizobium biofertilizer. p. 122-128. In S. Kannaiyan (ed.) Biotechnology of Biofertilizers. Narosa Publishing House, New Delhi, India.

Burd, G.I., D.G. Dixon and B.R. Glick. 2000. Plant growthpromoting bacteria that decrease heavy metal toxicity in plants. Canadian Journal of Microbiology 46: 23745.

Callan, N.W., D.E. Mathre and J.B. Miller. 1990. Biopriming seed treatment for control of Pythium ultimum pre emergence damping-off in sh-2 sweet corn. Plant Disease 74: 368-72.

Campbell, R., and Y.M. Ephgrave. 1983. Effect of bentonite clay on the growth of Gaeumannomyces graminis var. tritici and its interactions with antagonistic bacteria. Journal of General Microbiology 129: 771-777.

Chao, W.L. and M. Alexander. 1984. Mineral soils as carriers for Rhizobium inoculants. Applied Environmental Microbiology 47: 94-97.

Chi, F., S. Shen, H. Cheng, Y. Jing, Y.G. Yanni and F.B. Dazzo. 2005. Ascending migration of endophytic rhizobia, from roots to leaves, inside rice plants and assessment of benefits to rice growth physiology. Applied Environmental Microbiology 71: 7271-7278.

Dey, R., K.K. Pal, D.M. Bhatt and S.M. Chauhan. 2004. Growth promotion and yield enhancement of peanut (Arachis hypogaea L.) by application of plant growthpromoting rhizobacteria. Microbiological Research 159: 371-94.

Dworkin, M. and J. Foster. 1958. Experiments with some microorganisms which utilize ethane and hydrogen. Journal of Bacteriology. 75: 592-601.

Elegba, M.S. and R.J. Rennie. 1984. Effect of different inoculant adhesive agents on rhizobial survival, nodulation, and nitrogenase (acetylene-reducing) activity of soybeans (Glycine $\max$ (L.) Merrill). Canadian Journal of Soil Science 64: 631-636.

Frankenberger Jr, W.T. and M. Arshad. 1995. Phytohormones in Soil: Microbial Production and Function, Marcel Dekker, New York, USA.
Hebbar, P., A.G. Davey, J. Merrin, T.J. McLoughlin and P.J. Dart. 1992. Pseudomonas cepacia, a potential suppressor of maize soil borne diseases: seed inoculation and maize root colonization. Soil Biology and Biochemistry 24: 999-1007.

Herrera, M.A., C.P. Salamanka and J.M. Barea. 1993. Inoculation of woody legumes with selected arbuscular mycorrhizal fungi and rhizobia to recover desertified Mediterranean ecosystems. Applied Environmental Microbiology 59: 129-33.

Hussain, M.B., Z. A. Zahir, H. N. Asghar and S. Mahmood. 2014. Scrutinizing rhizobia to rescue maize growth under reduced water conditions. Soil Science Society of America Journal 78: 538-545.

Hussain, M.B., Z.A. Zahir, H.N. Asghar, R. Mubaraka and M. Naveed. 2016. Efficacy of rhizobia for improving photosynthesis, productivity and mineral nutrition of maize. CLEAN - Soil, Air, Water 44: 1564-1571.

Hussain, S., M.B. Hussain, A. Gulzar, M. Zafar-ul-Hye, M. Aon, M. Qaswar and M. Rizwan. 2017. Time of zinc and phosphorus applications to maize is depended on nutrient-nutrient and nutrient-inoculation interactions. Soil Science and Plant Nutrition. DOI: 10.1080/00380768.2017.1361784

Islam, F., T. Yasmeen, Q. Ali, S. Ali, M.S. Arif, S. Hussain and H. Rizvi. 2014. Influence of Pseudomonas aeruginosa as PGPR on oxidative stress tolerance in wheat under $\mathrm{Zn}$ stress. Ecotoxicology and Environmental Safety 104: 285-293.

Ryan, J. G. Estefan and A. Rashid. 2001. Soil and Plant Analysis Laboratory Manual, Jointly published by the International Centre Agricultural Research in Dry Areas (ICARDA), Aleppo, Syria and National Agricultural Research Centre (NARC), Islamabad.

Jackson, M.L. 1962. Chemical composition of soil. p. 71144. In F.E. Bean (ed.) Chemistry of Soil. Van Nostrand Co., New York.

Kloepper, J.W. and M.N. Schroth. 1978. Plant growth promoting rhizobacteria on radishes. p. 879-82. In Proceedings of the Fourth International Conference on Plant Pathogen Bacteria, Vol. 2. INRA, Gilbert-Clarey, Tours, France.

Kloepper, J.W., J. Leong, M. Teintze and M.N. Schroth. 1980. Pseudomonas siderophores: A mechanism explaining disease suppressive soils. Current Microbiology 4: 317-320.

Liu, L., J.W. Kloepper and S. Tuzun, Induction of systemic resistance in cucumber against fusarium wilt by plant growth-promoting rhizobacteria. Phytopathology 85: 695-698.

Mahmood, A., O.C. Turgay, M. Farooq and R. Hayat. 2016. Seed biopriming with plant growth promoting 
rhizobacteria: a review. FEMS Microbiology Ecology. doi: 10.1093/femsec/fiw112

Mehboob, I., Z.A. Zahir, M. Arshad, A. Tanveer and M. Khalid. 2012. Comparative effectiveness of different rhizobium sp. for improving growth and yield of maize (Zea mays L.), Soil and Environment 31: 37-46.

Meyer, J.M. 2000. Pyoverdines: Pigments, siderophores and potential taxonomic markers of fluorescent Pseudomonas sp. Archives of Microbiology 174: 135142.

Milus, E.A. and C.S. Rothrock. 1993. Rhizosphere colonization of wheat by selected soil bacteria over diverse environments. Canadian Journal of Microbiology 39: 335-341.

Nadeem, S.M., Z.A. Zahir, M. Naveed and S. Nawaz. 2013. Mitigation of salinity induced negative impact on the growth and yield of wheat by plant growth-promoting rhizobacteria in naturally saline conditions. Annals of Microbiology 63: 225-232.

Nadeem, S.M., Z.A. Zahir, M. Naveed, H.N. Asghar and M. Arshad. 2010. Rhizobacteria capable of producing ACC-deaminase may mitigate salt stress in wheat. Soil Science Society of America Journal 74: 533-542.

Naveed, M., M.A. Qureshi, Z.A. Zahir, M.B. Hussain, A. Sessitsch and B. Mitter. 2014a. L-Tryptophan dependent biosynthesis of indole-3-acetic acid (IAA) improves plant growth promotion and colonization of maize by Burkholderia phytofirmans PsJN. Annals of Microbiology 65: 1381-1389.

Naveed, M., M.B. Hussain, Z.A. Zahir, B. Mitter and A. Sessitsch. 2014b. Drought stress amelioration in wheat through inoculation with Burkholderia phytofirmans strain PsJN. Plant Growth Regulation 73: 121-131.

Neeraja, C., K. Anil, P. Purushotham, K. Suma, P. Sarma, B.M. Moerschbacher and A.R. Podile. 2010. Biotechnological approaches to develop bacterial chitinases as a bioshield against fungal diseases of plants. Critical Reviews in Biotechnology 30: 231-241.

Paulucci, N.S., L.A. Gallarato, Y.B. Reguera, J.C. Vicario, A.B. Cesari, M.B. García de Lema and M.S. Dardanelli. 2015. Arachis hypogaea PGPR isolated from Argentine soil modifies its lipids components in response to temperature and salinity. Microbiological Research 173: 1-9.

Podile, A.R. and G.K. Kishore. 2006. Plant growth promoting rhizobacteria. p. 195-230. In S.S. Gnanamanickam (ed.). Plant Associated Bacteria. Dordrecht, Springer, Netherlands.

Principe, A., F. Alvarez, M.G. Castro, L. Zachi, S.E. Fischer, G.B. Mori and E. Jofre. 2007. Biocontrol and PGPR features in native strains isolated from saline soils of Argentina. Current Microbiology 55: 314-322.
Requena, B.N., I. Jimenez, M. Toro and J.M. Barea. 1997. Interaction between plant growth promoting rhizobacteria (PGPR) arbuscular fungi and Rhizobium spp. in the rhizosphere of Anthyllis cytiisoides, a model legume for regeneration in Mediterranean semi-arid ecosystem. New Phytologist 136: 667-677.

Roy, M. and P.S. Basu. 2004. Studies on root nodules of leguminous plants bioproduction of indole acetic acid by a Rhizobium sp. from a twiner Clitoria ternatea $\mathrm{L}$. Acta Biotechnology 12: 453-460.

Saravanakumar, D., N. Lavanya, B. Muthumeena, T. Raguchander, S. Suresh and R. Samiyappan. 2008. Pseudomonas fluorescens enhances resistance and natural enemy population in rice plants against leaf folder pest. Journal of Applied Entomology 132: 469479.

Sarma, M.V.R.K., V. Kumar, K. Saharan, R. Srivastava, A.K. Sharma, A. Prakash, V. Sahai and V.S. Bisaria. 2011. Application of inorganic carrier-based formulations of fluorescent pseudomonads and Piriformospora indica on tomato plants and evaluation of their efficacy. Journal of Applied Microbiology 111: 456-466.

Shahzad, S., M.Y. Khan, Z.A. Zahir, H.N. Asghar and U.K. Chaudhry. 2017. Comparative effectiveness of different carriers to improve the efficacy of bacterial consortium for enhancing wheat production under salt affected field conditions. Pakistan Journal of Botany 49: 1523-1530.

Staley, T.E. and D.J. Drahos. 1994. Marking soil bacteria with lacZY. p. 689-706. In R.W. Weaver, J.S. Angel and P.J. Bottomley (eds) Methods of Soil Analysis. Part 2. Microbiological and Biochemical Properties. Soil Science Society of America, Madison, WI.

Steel, R.G.D., J.H. Torrie and D.A. Dicky. 1997. Principles and Procedures of Statistics- A Biometrical Approach. (3rd Edn.). P. 204-227. McGraw Hill Book International Co., Singapore.

Taylor, A.G. and G.E. Harman. 1990. Concept and technologies of selected seed treatments. Annual Review of Phytopathology 28: 321-339.

Upadhyaya, N.M., D.S. Letham, C.W. Parker, C.H. Hocart and P.J. Dart. 1991. Do rhizobia produce cytokinins? Biochemistry International 24: 123-130.

Wolf, B. 1982. The comprehensive system of leaf analysis and its use for diagnosting crop nutrient status. Communications Soil Science and Plant Analysis 13: 1035-1059.

Zaidi, S., S. Usmani, B.R. Singh and J. Musarrat. 2006. Significance of Bacillus subtilis strain SJ-101 as a bioinoculant for concurrent plant growth promotion and nickel accumulation in Brassica juncea. Chemosphere 64: 991-997. 